\title{
A Holistic Conception of Electric Mobility
}

\section{Dear Reader,}

The journey is the destination; or the car is the grid. In the case of electric mobility, joining the two is a central concern. Whether you're a skeptic in matters of supply security or an optimist who thinks everything will continue to work smoothly, it's not going to happen without grid integration. And this is contrary to the current status quo where vehicles and fueling stations are extensively decoupled.

On the one hand, a critical mass of electric vehicles combined with an increasing share of less secure sources of renewable energy introduces new grid-control challenges whose resolution will require considerable efforts. On the other hand, we have an opportunity to change our tendency to regard electric vehicles as isolated, self-contained systems. And here, it's no surprise that Tesla has taken the lead. The California-based company ticks differently than the classic carmakers, which is perhaps to be expected as building cars isn't where Tesla primarily makes its money. As has been reported by Manager Magazin Online and others, Tesla's concept is supported by multiple pillars: acting as an authorized supplier of electricity, operating a factory in Brandenburg as a direct customer, and deploying a vehicle fleet and Powerwall home energy units as a buffer. Given the periodic availability of free or even paid surplus power on the market, there is potential there that other carmakers aren't in a position to exploit.

The example of Tesla shows how enterprises can expand their business models by understanding and engaging promis- ing peripheral domains instead of merely attending to sharply demarcated products. What could be really exciting is that Tesla might be able to supply its own vehicle with power at favorable rates at its own charging stations while also including them as participants on the power-supply market by bundling their vehicles to create a buffer on the grid. While the current total of approximately 20,000 registered Tesla vehicles is perhaps still in the negligible range, the scenario could change dramatically in the wake of programs to incentivize the purchase of electric vehicles. That would certainly be a boon for the company as the provider, but also very likely for Tesla customers and for the grid as a whole, as a "Giga-battery" is attached to it much sooner than planned.

I hope you enjoy reading this latest issue!

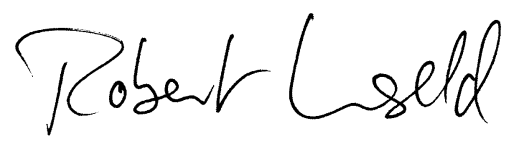

Robert Unseld Responsible Editor

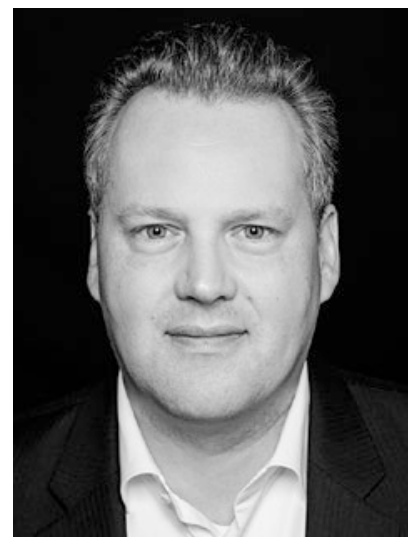

